\title{
The Statistical Analysis of Pharmacokinetic Param- eters in the Context of Bioequivalence Testing of Two Anthelmintic Formulas Based on Ivermectine and Triclabendazole in Sheep
} \author{
Andreea Buta ${ }^{4}$, Laurentiu Ognean ${ }^{4}$ \\ 1. University of Medicine, Pharmacy, Science and Technology of Targu Mures, Romania \\ 2. "Iuliu Hațieganu" University of Medicine and Pharmacy, Cluj-Napoca, Romania \\ 3. Clinical and Analytical Research Center Vim Spectrum \\ 4. University of Agricultural Science and Veterinary Medicine Cluj-Napoca, Romania
}

Lenard Farczadi ${ }^{1}$, Laurian Vlase ${ }^{\star}$, Orsolya Melles ${ }^{3}$, Ramona Tolomeiu4, Octavia Tamas-Krumpe ${ }^{4}$,

Conducting bioequivalence studies is an essential step during the market authorization process of generic pharmaceutical formulations, for both human or veterinary use. The aim of the present study was to evaluate the pharmacokinetics of triclabendazole sulphoxide, the main metabolite of triclabendazole, and ivermectin in order to evaluate the bioavailability and bioequivalence of a novel sheep anthelmintic formulation of oral suspension for sheep treatment containing triclabendazole $50 \mathrm{mg} / \mathrm{mL}$ and ivermectin $1 \mathrm{mg} / \mathrm{mL}$ compared to the reference product. In order to determine relative bioavailability of the test product with respect to the reference product the study was conducted on 36 clinically healthy sheep, following an unicentric, randomized, cross-over, two-sequence, two-treatment and 14-day wash-out study design. For the determination of triclabendazole sulphoxide and ivermectin sheep plasma concentrations, two rapid, selective high performance liquid chromatography coupled with mass spectrometry (LC-MS/MS) methods were developed and validated. The measured plasma concentrations of triclabendazole sulphoxide and ivermectin were used for the pharmacokinetic analysis and the determination of bioequivalence between the test product with regards to the reference product. The noncompartmental analysis of the pharmacokinetic data for both triclabendazole sulphoxide and ivermectin showed similarities between first-order kinetics of the test and reference product. The relevant pharmacokinetic parameters (Cmax, AUClast, AUCtot) were determined and the bioequivalence between the test and reference product could be concluded.

Keywords: ivermectin, triclabendazole, pharmacokinetics, bioequivalence

Received 2 May 2019 / Accepted 14 June 2019

\section{Introduction}

Triclabendazole is an anthelmintic drug which is used for the treatment of liver fluke, Fasciola hepatica and Fasciola gigantica, in sheep and other livestock. Triclabendazole cannot only be used for treating liver fluke in livestock but may also be used in cases of human fascioliasis [1].

Following oral administration and absorption triclabendazole is rapidly metabolized in the liver. Triclabendazole and its two metabolites, triclabendazole sulphoxide and triclabendazole sulphone, have high activity against the migratory juvenile stages of liver fluke. Both metabolites show important plasma protein binding which explains their relatively slow elimination from the animal's organism. Triclabendazole sulphoxide and triclabendazole sulphone are also the main two unconjugated metabolites present in bile. The metabolic mechanism of triclabendazole is complex, but helps maintaining concentrations levels of triclabendazole and its metabolites in the bloodstream over longer periods of time which in turn contributes to its high efficacy against the fluke [2].

Triclabendazole sulphoxide is the main active metabolite of triclabendazole and the major metabolite detected

* Correspondence to: Laurian Vlase

E-mail: laurian.vlase@umfcluj.ro in the host [2]. Thus, the bioequivalence of a product containing triclabendazole can be evaluated by analyzing the pharmacokinetics of the metabolite in plasma and comparing it between the generic (test) and reference product.

Ivermectin is a mixture of two chemically modified avermectins (B1a and B1b) and it is a very widely used drug against a wide array of nematode species. It is lipophilic and thus easily soluble in organic solvents but not water soluble [3]. Due to this it tends to accumulate in fat tissue and persist for longer periods of time in the body not only due to this accumulation but also due to its low plasma clearance. For bioequivalence purposes the pharmacokinetics of avermectin B1a in plasma were analyzed and compared between the test and reference product, as avermectin B1a makes up more than $90 \%$ of ivermectin.

In order to further extend the spectrum of use, the antiparasitic drugs are often used in combined therapy [4]. In some cases, the combined therapy may even yield higher efficacy than the separately applied treatments [5]. Due to the multiple compounds, however, the bioequivalence studies performed on combined products are more difficult and complex, from both analytical point of view as well as due to the increased number of pharmacokinetic analyzes which need to be carried out. 
Bioequivalence studies are an essential step in the process of obtaining marketing authorization for generic medicinal products in the USA and Europe. As at the time of the study no generic products containing a combination of triclabendazole and ivermectin were marketed, the launch of a generic product is very important. Generic medicinal products not only offer consumers a choice, but can also satisfy demand in areas where the originator product is scarcely available or not available. On the other hand due to competition the authorization of generic product leads to a decrease in prices and makes products more accessible to a larger population, especially in underdeveloped countries

The aim of the present study was to evaluate the pharmacokinetics of triclabendazole sulphoxide, the main metabolite of triclabendazole, and ivermectin in order to evaluate the bioavailability and bioequivalence of a novel sheep anthelmintic formulation, an oral suspension containing triclabendazole $50 \mathrm{mg} / \mathrm{ml}$ and ivermectin $1 \mathrm{mg} / \mathrm{ml}$, compared to the reference product.

\section{Methods}

\section{Subjects}

The study was conducted on a total of 36 healthy male and female sheep in accordance with the following guidelines: Good Clinical Practice guidelines [6], EMA Guidelines for the Conduct of Bioequivalence Studies for Veterinary Medicinal Products $[7,8,9]$ as well as other applicable regulations or laws enforceable in Romania.

The study protocol was reviewed and approved by the Ethics Committee of the University of Agricultural Sciences and Veterinary Medicine Cluj-Napoca.

Sheep were deemed to be healthy judged by means of a medical and standard laboratory examination (normal hematology, clinical chemistry and urinalysis).

\section{Study design}

The study consisted of two study periods. All animals received in each of both periods of the study a single dose of product, $0.6 \mathrm{ml} / \mathrm{kg}$ bodyweight (either test or reference formulation). The first period of the study was followed, after a 14 days washout period, by the second period of the trial. The sheep were fast from food from the morning of the day of the drug administration until 24 hours after administration. Water was allowed ad libitum until 2 hours before and 2 hours after administration. Products administered were the test product Trimectin $50 \mathrm{mg} / \mathrm{ml}+$ $1 \mathrm{mg} / \mathrm{ml}$ Oral Suspension for Sheep (ivermectin $1 \mathrm{mg}$, triclabendazole $50 \mathrm{mg}$ ) (Pharma VIM Kft., Hungary) and the reference product Fasimec Duo $50 \mathrm{mg} / \mathrm{ml}+1 \mathrm{mg} / \mathrm{ml}$ Oral Suspension for Sheep (ivermectin $1 \mathrm{mg}$, triclabendazole 50 mg) (Elanco Animal Health, UK).

Venous blood samples $(5 \mathrm{~mL})$ were drawn from the jugular vein, from the neck area, of each subject in tubes containing anticoagulant (K3EDTA) before the administration (time 0.0 ) and at $0.5 ; 1.0 ; 1.5 ; 2.0 ; 3.0 ; 4.0 ; 6.0$; $8.0 ; 10.0 ; 12.0 ; 14.0 ; 16.0 ; 18.0 ; 20.0 ; 22.0 ; 24.0 ; 28.0$; $36.0 ; 48.0 ; 72.0 ; 96.0 ; 120.0 ; 144.0 ; 168.0 ; 216.0 ; 264.0$; $336.0 ; 408.0 ; 480.0$ hours post dose administration. Samples were centrifuged using a Centurion Scientific K241R Centrifuge, at $5000 \mathrm{rpm}$ for 10 minutes within 60 minutes after collection. The plasma was separated and transferred to two test tubes (sample and backup sample). The test tubes were securely closed, labeled and immediately frozen for storage at $-20^{\circ} \mathrm{C}$ until analysis.

\section{Analysis of plasma samples}

Sheep plasma concentrations of triclabendazole sulphoxide and ivermectin (avermectin B1a) were determined using validated high-performance liquid chromatography coupled with mass spectrometry (LC-MS/MS) methods which were developed in-house. A typical chromatogram for triclabendazole sulphoxide and its internal standard are presented in Figure 1.

\section{Pharmacokinetic and statistical analysis}

Data from all evaluable subjects were included in bioequivalence assessment. All 36 subjects finalized the study and
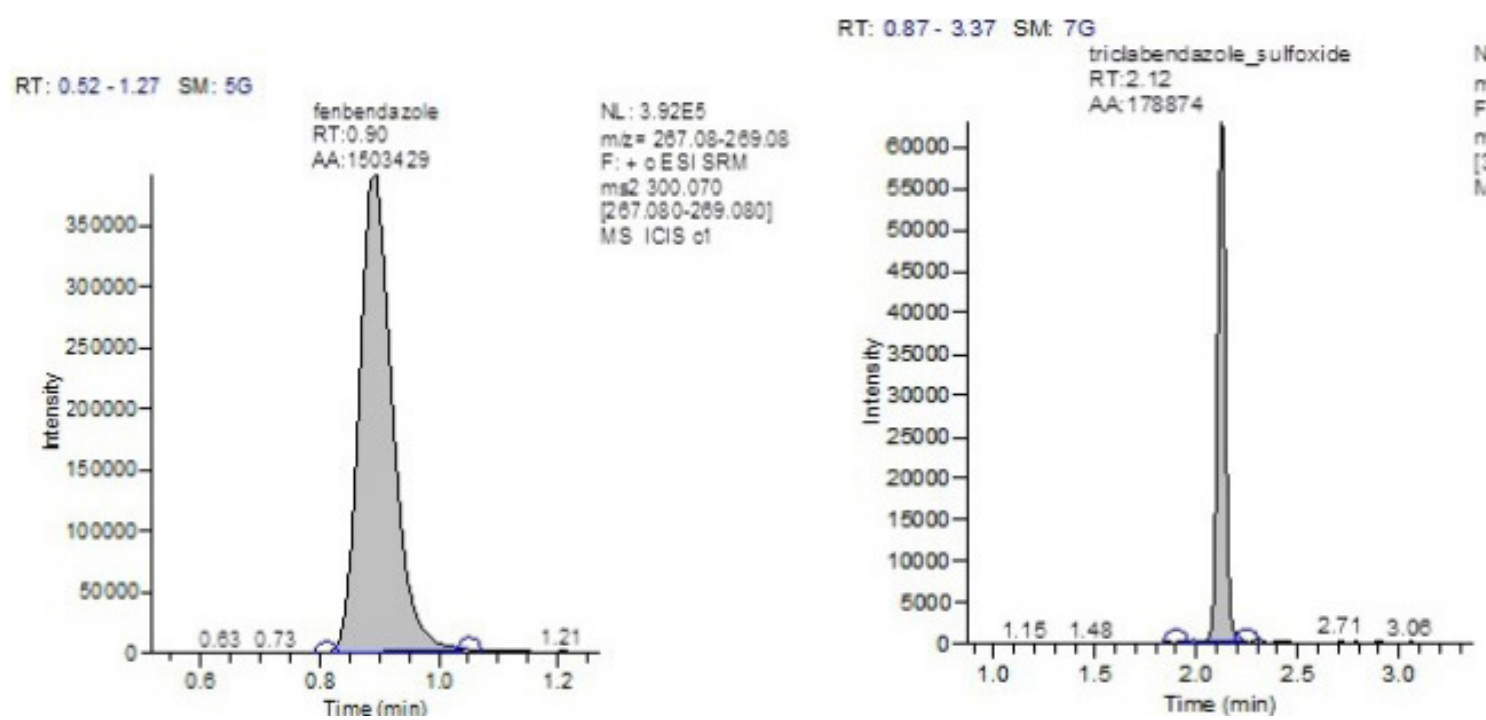

Fig. 1. A typical chromatogram for triclabendazole sulphoxide and its internal standard (fenbendazole) 
were included in the statistical analysis of pharmacokinetic data. Noncompartmental pharmacokinetic analysis method was carried out for both triclabendazole sulphoxide and ivermectin in order to determine the pharmacokinetic parameters. For bioequivalence assessment for all the primary pharmacokinetic parameters considered (Cmax, AUClast) a confidence interval of $90 \%$ for the ratio of the population means T/R (Test/Reference) was calculated. All these pharmacokinetic parameters were planned for analysis using ANOVA, after data logarithmic transformation. A reference $90 \%$ confidence interval of $0.8-1.25$ was chosen.

In addition, summary statistics were performed, including arithmetic mean, harmonic mean, geometric mean, SEM, standard deviation, median, range. For Tmax comparison, non-parametric tests were carried out (KruskalWallis and Friedman test) on untransformed data. For MRT (mean residence time) and Thalf the same statistical tests were applied as for primary parameters (Latin-square ANOVA with determination of $90 \%$ CI of the ratio of means $T / R$ after data log-transformation and descriptive statistics). The pharmacokinetic analysis was performed using Kinetica 5 (ThermoLabsystems, USA) [10].

In order to evaluate a possible statistical or clinical significance of the pharmacokinetic interaction, an analysis of variance (ANOVA) was performed on the main calculated pharmacokinetic parameters, using general linear model procedures, in which the sources of variation were the subject and the treatment. All calculations and evaluations were analyzed taking into consideration the current applicable guidelines $[11,12]$.

\section{Results}

All sheep plasma samples obtained during the study were analyzed using the fully validated LC-MS method developed in-house. Improbable results obtained during initial assay of samples were reanalyzed in accordance with the current guidelines and incurred samples were also analyzed on the last day of analysis as part of the validation process.
During incurred samples analysis the percent difference between concentrations obtained for the initial analysis and the concentrations obtained after reanalysis was not greater than $20 \%$ of their mean for $74 \%$ of samples for triclabendazol sulphoxide and $84 \%$ of samples for ivermectin, respectively.

The mean plasma concentration curves for triclabendazole sulphoxide and ivermectin were determined, for both test and reference product, and are shown in Figures 2, 3, 4 and 5 respectively.

The mean values for triclabendazole sulphoxide Cmax were $56.0(+/-17.1) \mu \mathrm{g} / \mathrm{ml}$ for test and $54.4(+/-20.1) \mu \mathrm{g} /$ $\mathrm{ml}$ for the reference product. For ivermectin Cmax mean values were $41.2(+/-8.7) \mathrm{ng} / \mathrm{ml}$ for test and $42.2(+/-10.5)$ $\mathrm{ng} / \mathrm{ml}$ for reference product, respectively.

Statistical analysis was carried out for the obtained data. After applying ANOVA the $90 \%$ confidence intervals for the ratio of means of triclabendazole sulphoxide and ivermectin "Test/Reference" for the highest concentrations (Cmax) were 0.98-1.12 for triclabendazol sulphoxide and 0.92-1.05 for ivermectin. The $90 \%$ confidence intervals for the ratio of means of triclabendazole sulphoxide and ivermectin "Test/Reference" for the area under the curve up to the last measurable concentration (AUClast) were 0.88-1.07 for triclabendazole sulphoxide and 0.86-1.06 for ivermectin. The mean pharmacokinetic parameters for triclabendazole sulphoxide and ivermectin as well as the statistics for them are shown in Table I and Table II.

\section{Discussions}

Incurred sample reanalysis testing passed criteria stipulated in current bioanalytical method validation guidelines $[13,14]$ as the percent difference between concentrations obtained for the initial analysis and the concentrations obtained after reanalysis was within the $+/-20 \%$ of their mean for more than $67 \%$ of the reanalyzed samples, for each triclabendazol sulphoxide and ivermectin. Thus, the LC-MS method used for analysis being validated and in-

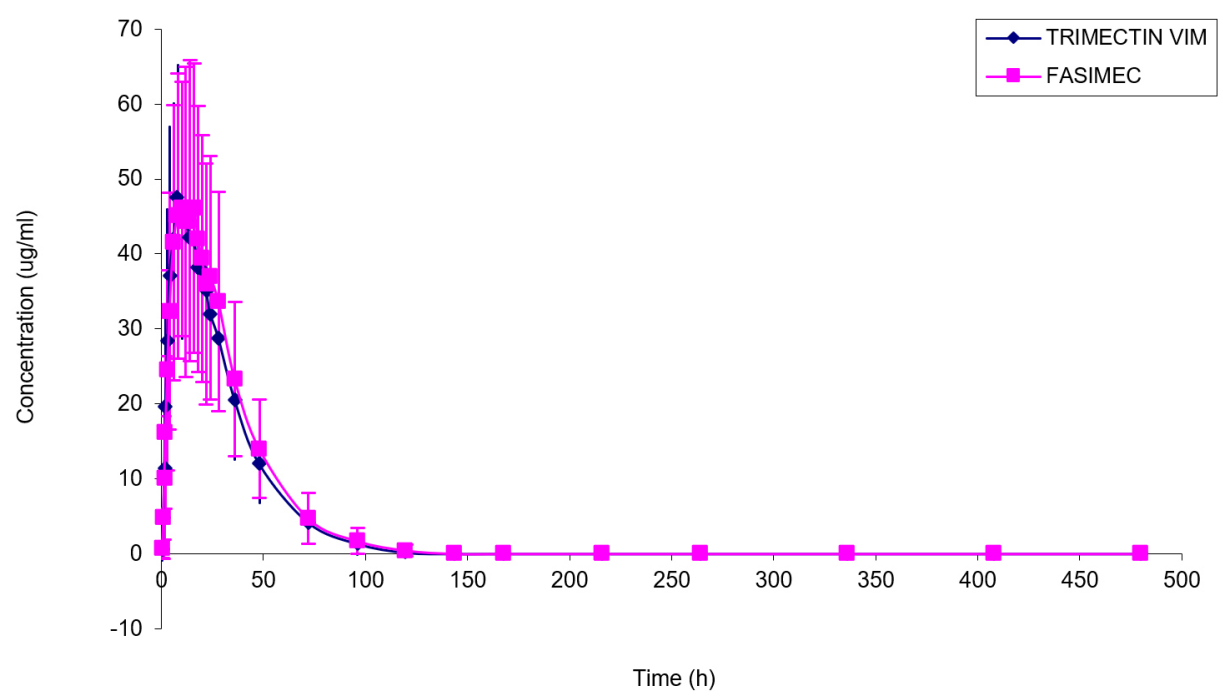

Fig. 2. Mean plasma concentration curves for triclabendzole sulphoxide in bioequivalence testing of two anthelmintic formulas for sheep 


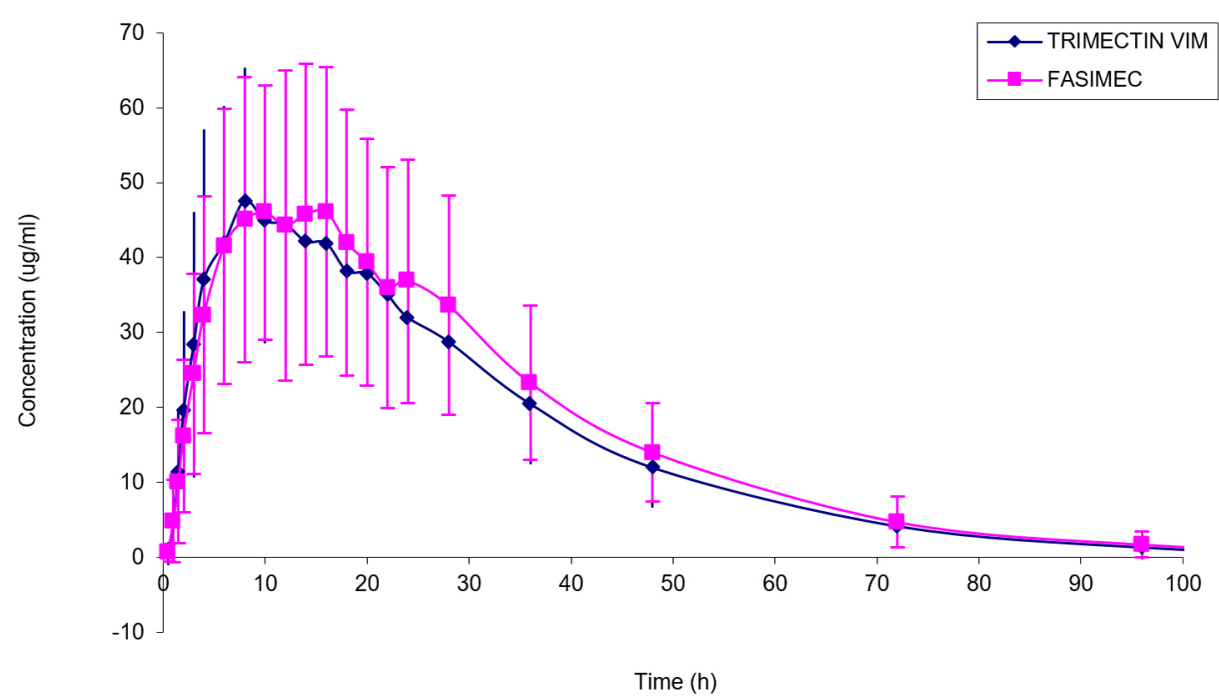

Fig. 3. Mean plasma concentration curves for triclabendzole sulphoxide in bioequivalence testing of two anthelmintic formulas for sheep, zoomed in for the interval $0-100 \mathrm{~h}$

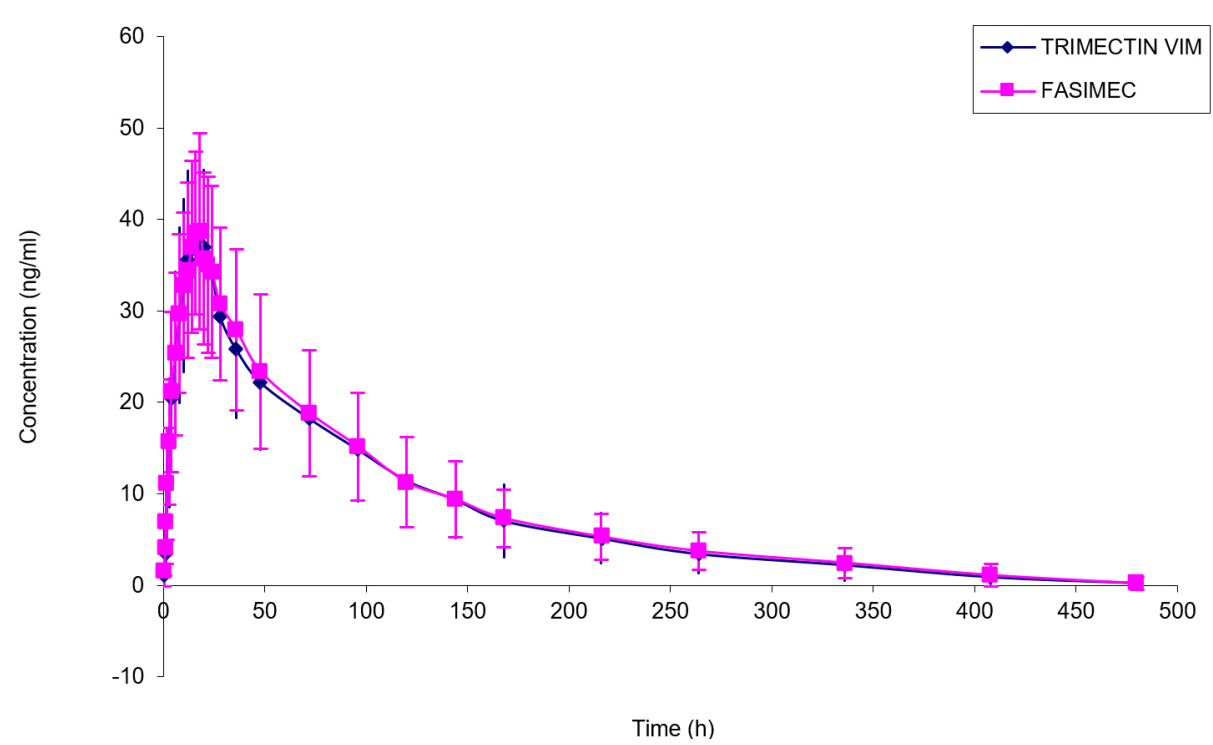

Fig. 4. Mean plasma concentration curves for ivermectin in bioequivalence testing of two anthelmintic formulas for sheep

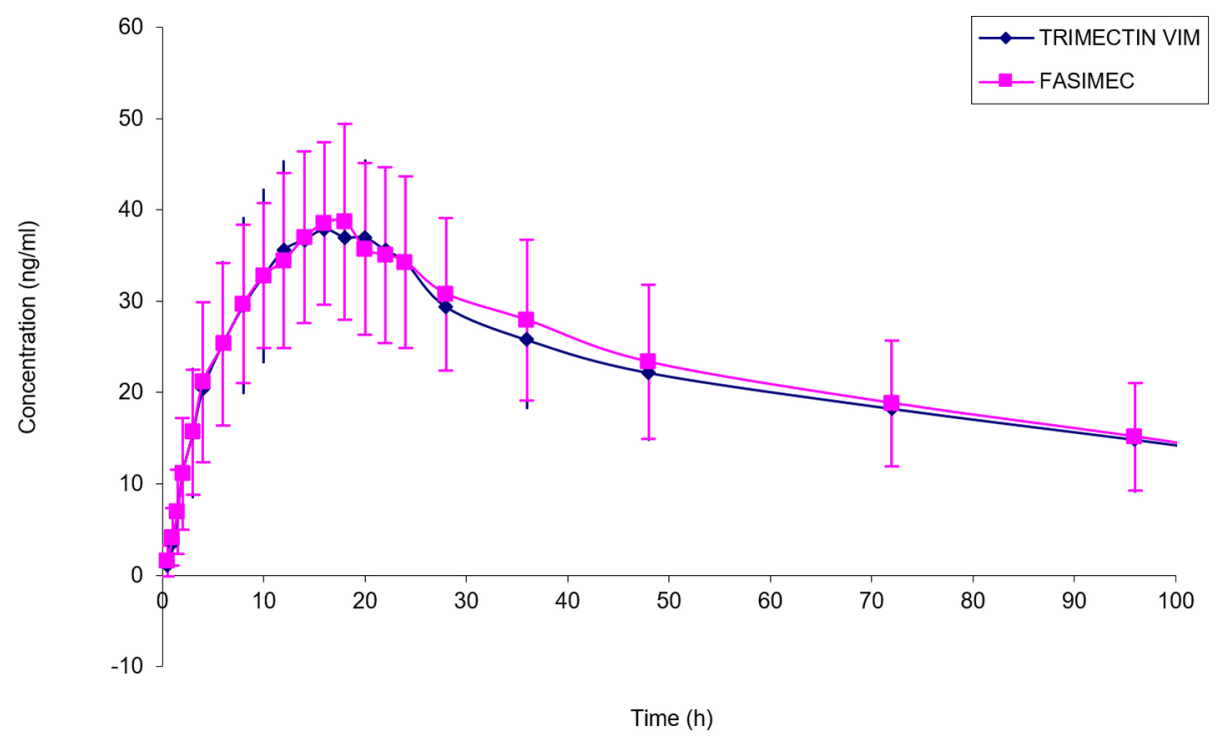

Fig. 5. Mean plasma concentration curves for ivermectin in bioequivalence testing of two anthelmintic formulas for sheep, zoomed in for the interval 0-100 h 
Table I. Statistics of primary, secondary and additional pharmacokinetic parameters of triclabendazole sulphoxide in test and reference groups

\begin{tabular}{|c|c|c|c|c|c|c|c|}
\hline \multirow[t]{2}{*}{ Parameter } & \multicolumn{2}{|c|}{$\begin{array}{l}\text { TEST } \\
\text { Trimectin }\end{array}$} & \multicolumn{2}{|c|}{$\begin{array}{l}\text { REFERENCE } \\
\text { Fasimec Duo }\end{array}$} & \multirow{2}{*}{$\begin{array}{c}\text { Geometric } \\
\text { mean ratio T/R }\end{array}$} & \multirow{2}{*}{$\begin{array}{l}p^{*} \text { value } \\
\text { ANOVA }\end{array}$} & \multirow{2}{*}{$\begin{array}{c}90 \% \text { confidence interval } \\
(\%)\end{array}$} \\
\hline & Mean & SD & Mean & SD & & & \\
\hline Cmax $(\mu \mathrm{g} / \mathrm{ml})$ & 55.997 & 17.124 & 54.402 & 20.100 & 1.05119 & $0.2184 \mathrm{NS}$ & $0.98-1.12$ (ANOVA, NS) \\
\hline $\operatorname{Tmax}(\mathrm{h})$ & 11.583 & 5.6334 & 11.833 & 4.6935 & 0.978873 & - & $\chi^{2}=3.841$ (Fried, K-W, NS) \\
\hline AUClast (ng/ml*h) & 1655.6 & 443.85 & 1803.3 & 750.6 & 0.969058 & $0.5863 \mathrm{NS}$ & $0.88-1.07$ (ANOVA, NS) \\
\hline AUCtot (ng/ml*h) & 1702.4 & 445.88 & 1847.7 & 755.64 & 0.970008 & $0.5870 \mathrm{NS}$ & - \\
\hline Thalf (h) & 15.517 & 3.7018 & 15.594 & 3.5047 & - & $0.8828 \mathrm{NS}$ & - \\
\hline MRT (h) & 28.334 & 5.5840 & 29.388 & 4.5714 & - & $0.1017 \mathrm{NS}$ & - \\
\hline
\end{tabular}

Table II. Statistics of primary, secondary and additional pharmacokinetic parameters of ivermectin in test and reference groups

\begin{tabular}{|c|c|c|c|c|c|c|c|}
\hline \multirow{2}{*}{ Parameter } & \multicolumn{2}{|c|}{$\begin{array}{c}\text { TEST } \\
\text { Trimectin }\end{array}$} & \multicolumn{2}{|c|}{$\begin{array}{l}\text { REFERENCE } \\
\text { Fasimec Duo }\end{array}$} & \multirow{2}{*}{$\begin{array}{c}\text { Geometric } \\
\text { mean ratio T/R }\end{array}$} & \multirow{2}{*}{$\begin{array}{l}p^{*} \text { value } \\
\text { ANOVA }\end{array}$} & \multirow{2}{*}{$\begin{array}{c}90 \% \text { confidence interval } \\
(\%)\end{array}$} \\
\hline & Mean & SD & Mean & SD & & & \\
\hline Cmax $(\mu \mathrm{g} / \mathrm{ml})$ & 41.220 & 8.6637 & 42.182 & 10.462 & 0.981343 & $0.6191 \mathrm{NS}$ & $0.92-1.05$ (ANOVA, NS) \\
\hline $\operatorname{Tmax}(\mathrm{h})$ & 17.500 & 4.0107 & 18.333 & 5.6061 & 0.954545 & - & $\chi^{2}=3.841($ Fried, K-W, NS) \\
\hline AUClast (ng/ml*h) & 3804.3 & 1295.3 & 3926.9 & 1310.5 & 0.957828 & $0.4913 \mathrm{NS}$ & $0.86-1.06$ (ANOVA, NS) \\
\hline AUCtot (ng/ml*h) & 4001.0 & 1338.7 & 4172.6 & 1389.2 & 0.949778 & $0.4068 \mathrm{NS}$ & - \\
\hline Thalf (h) & 84.295 & 27.945 & 91.045 & 27.042 & - & $0.1977 \mathrm{NS}$ & - \\
\hline MRT (h) & 118.360 & 30.72 & 124.970 & 29.278 & - & $0.1901 \mathrm{NS}$ & - \\
\hline
\end{tabular}

curred sample analysis testing passing acceptance criteria, the plasma concentrations determined for the study samples can be considered accurate.

Based on the obtained plasma concentrations statistical analysis of data was carried out. The primary pharmacokinetic parameters (Cmax, Tmax, AUClast) were calculated for triclabendazol sulphoxide and ivermectin and used for the bioequivalence evaluation of the test product with regards to the reference product.

The parametric $90 \%$ confidence interval for the ratio $T / R$ period of the mean pharmacokinetic and the significance of the difference of Tmax values based on Friedman and Kruskal-Wallis test are also shown.

The $90 \%$ confidence intervals for the ratio of means of triclabendazole sulphoxide and ivermectin "Test/Reference" respectively, were within the conventional bioequivalence range of $80-125 \%$ for all primary parameters. The difference between means is not statistically significant for the Tmax of the test and reference products (Friedman and Kruskal-Wallis test) for neither triclabendazole sulphoxide nor ivermectin.

Thus, we were able to consider that all criteria described in current guidelines for bioequivalence testing were met for the test product $[11,12]$.

\section{Conclusion}

The bioequivalence could be concluded between the studied test and reference products due to the calculated $90 \%$ confidence interval around the ratio of means (Test/Reference) of $\log$ transformed data falling within the reference acceptance range for bioequivalence of $0.8-1.25$ for all primary pharmacokinetic parameters of both triclabenda- zole sulphoxide and ivermectin. The Friedman and Kruskal-Wallis tests showing no significant difference between Tmax of reference and test products for both triclabendazole sulphoxide and ivermectin.

\section{Acknowledgments}

The development of the analytical methods used for sample was financed by a grant of The Romanian National Authority for Scientific Research, CNCS-UEFISCDI, project number PN-III-P2-2.1-BG-2016-0249, project code BG113, competition BRIDGE 2016.

The main sponsor of the study was SC Vim Spectrum SRL Romania.

\section{Authors' contribution}

Lenard Farczadi (Formal analysis; Methodology; Validation; Writing - original draft)

Laurian Vlase (Methodology; Supervision; Writing - review $\&$ editing)

Orsolya Melles (Formal analysis; Project administration)

Ramona Tolomeiu (Investigation)

Octavia Tamas-Krumpe (Investigation)

Andreea Buta (Investigation)

Laurentiu Ognean (Conceptualization; Investigation; Supervision)

\section{References}

1. Barrera B, Otero JA, Egido E et al. The Anthelmintic Triclabendazole and Its Metabolites Inhibit the Membrane Transporter ABCG2/BCRP. Antimicrob Agents Chemother 2012; 56(7): 3535-3543.

2. Halferty L, Brennan GP, Trudgett A, Hoey L, Fairweather I. Relative activity of triclabendazole metabolites against the liver fluke, Fasciola hepatica. Vet Parasitol 2009; 159:126-138. 
3. González Canga A, Sahagún Prieto AM, José Diez Liébana M, Martínez NF, Vega MS, Vieitez JJ. The pharmacokinetics and metabolism of ivermectin in domestic animal species. Vet J 2009; 179: 25-37.

4. Lifschitz A, Virkel G, Ballent M, Sallovitz J, Lanusse C. Combined use of ivermectin and triclabendazole in sheep: In vitroand in vivo characterisation of their pharmacological interaction. Vet J 2009; 182: 261-268.

5. Stevenson CR, Mahoney RH, Fisara P, Strehlau G, Reichel MP. The efficacy of formulations of triclabendazole and ivermectin in combination against liver fluke (Fasciolahepatica) and gastro-intestinal nematodes in cattle andsheep and sucking lice species in cattle. Aust Vet J. 2002; 80(11): 698-701

6. International Committee for Harmonization. GUIDELINE FOR GOOD CLINICAL PRACTICE E6 (R1). https://www.ich.org/fileadmin/Public Web_Site/ICH_Products/Guidelines/Efficacy/E6/E6_R1_Guideline.pdf [retrieved March 22nd 2019]

7. The European Agency for the Evaluation of Medicinal Products. CVMP Guideline on the Conduct of Bioequivalence Studies for Veterinary Medicinal Products, EMA/CVMP/016/00-Rev.2.

8. https://www.ema.europa.eu/en/documents/scientific-guideline/ guideline-conduct-bioequivalence-studies-veterinary-medicinalproducts-revision-2_en.pdf [retrieved March 22nd 2019]

9. The European Agency for the Evaluation of Medicinal Products. CVMP Guidelines for the Conduct of Bioequivalence Studies for Veterinary Medicinal Products, EMEA/CVMP/016/00-corr-FINAL.

10. https://www.ema.europa.eu/en/documents/scientific-guideline/ guideline-conduct-bioequivalence-studies-veterinary-medicinalproducts-revision-1_en.pdf [retrieved March 22nd 2019]
11. The European Agency for the Evaluation of Medicinal Products. $\mathrm{VICH}$ GL52 Bioequivalence: Blood Level Bioequivalence Study, EMA/CMVP/ VICH/751935/2013-Corr. https://www.ema.europa.eu/en/documents/ scientific-guideline/international-cooperation-harmonisation-technicalrequirements-registration-veterinary-medicinal_en-3.pdf [retrieved March 22nd 2019]

12. http://kineticadownload.com/Kinetica5.0/data/Kinetica\%20User\%20 Manual.pdf [retrieved March 22nd 2019]

13. U.S. Department of Health and Human Services, Food and Drug Administration, Bioavailability and Bioequivalence Studies for Orally Administrated Drug Products - General Considerations, Rockville, USA, 2003, https://www.fda.gov/downloads/Drugs/Guidances/ucm154838 [retrieved March 22nd 2019]

14. The European Agency for the Evaluation of Medicinal Products. Note for Guidance on the Investigation of Bioavailability and Bioequivalence, London, UK, 2001 (CPMP/EWP/QWP/1401/98). https://www.ema. europa.eu/en/documents/scientific-guideline/draft-note-guidanceinvestigation-bioavailability-bioequivalence_en.pdf [retrieved March 22nd 2019]

15. The European Agency for the Evaluation of Medicinal Products. Guideline on bioanalytical method validation (EMEA/CHMP/EWP/192217/2009 Rev. 1 Corr. 2). https://www.ema.europa.eu/en/documents/scientificguideline/guideline-bioanalytical-method-validation en.pdf [retrieved March 22nd 2019]

16. U.S. Department of Health and Human Services, Food and Drug Administration. Bioanalytical Method ValidationGuidance for Industry. https://www.fda.gov/downloads/Drugs/Guidances/ucm070107.pdf [retrieved March 22nd 2019] 